\title{
OBSERVATIONS OF VECTOR MAGNETIC FIELDS IN FLARING ACTIVE REGIONS
}

\author{
JIMIN CHEN* ${ }^{*}$ HAIMIN WANG, HAROLD ZIRIN \\ Big Bear Solar Observatory, California Institute of Technology, Pasadena, CA 91125, U.S.A. \\ and \\ GUOXIANG AI \\ Beijing Astronomical Observatory, Chinese Academy of Sciences, Beijing 100080, China
}

(Received 30 December, 1993; in revised form 12 July, 1994)

\begin{abstract}
We present vector magnetograph data of 6 active regions, all of which produced major flares. Of the $20 \mathrm{M}$-class (or above) flares, 7 satisfy the flare conditions prescribed by Hagyard (high shear and strong transverse fields). Strong photospheric shear, however, is not necessarily a condition for a flare. We find an increase in the shear for two flares, a 6-deg shear increase along the neutral line after a X-2 flare and a 13-deg increase after a M-1.9 flare. For other flares, we did not detect substantial shear changes.
\end{abstract}

\section{Introduction}

Hagyard (1992) studied the relationship between 4 flares and vector magnetic fields, and found the following results: (1) each of the four flares occurred in an area where the local photospheric magnetic field departed the most from a potential field; (2) the initial flare brightenings were located on either side of the neutral line near the point where the angular shear attained its greatest value; (3) a sufficient condition for flares to occur is that the maximum angular shear be at least $85^{\circ}$ and the interval along the neutral line in which the fields are strong and the angular shear remains high $\left(\geq 80^{\circ}\right)$ extend for $8000-10000 \mathrm{~km}$. Recently, Wang and colleagues (Wang, 1992; Wang and Tang, 1993; Wang et al., 1994) found that the photospheric magnetic shear increases after X-class flares. To investigate these problems further, in this paper, we will discuss the configurations of vector magnetic fields before 20 M-class (or above) flares and magnetic shear changes along the neutral lines before and after the flares.

\section{Data}

The photospheric vector magnetograms in this study were obtained by the Solar Magnetic Field Telescope (SMFT) at Huairou Observing Station of Beijing Astronomical Observatory. The SMFT system uses the Fe I 5324 line for the photospheric measurement (Ai, 1986). After 256-frame integration, the noise level of the lineof-sight magnetogram is $\sim 10 \mathrm{G}$; for the transverse magnetogram with the same

* Visiting Associate from Beijing Astronomical Observatory, Chinese Academy of Sciences, Beijing 100080, China.

(C) 1994 Kluwer Academic Publishers. Printed in Belgium. 
integration, it is $\sim 150 \mathrm{G}$. We plan to study all the M-class or above flares for which we have vector magnetograms before and after the flares. In this paper, we present the data of 1989 and 1990.

We use the same angular shear, $\theta$, defined by Hagyard (1984), which is the angle between the measured transverse field and the potential field derived by using the observed line-of-sight fields as the boundary conditions. To avoid the $180^{\circ}$ ambiguity of the observed azimuth, we restrict the shear angle to be $<90^{\circ}$.

To reduce the noise of weak transverse fields, we further define the 'mean weighted shear angle' as

$$
\bar{\Theta}=\frac{\sum\left|B_{t}\right| \theta}{\sum\left|B_{t}\right|}
$$

where $B_{t}$ is the measured transverse field strength (Wang, 1992).

\section{Results}

\subsection{CONFIGURATIONS OF VECTOR MAGNETIC FIELDS BEFORE THE FLARES}

We first present two flares which represent two typical situations: (1) a flare is not associated with strong magnetic shear, and (2) a flare is associated with strong magnetic shear.

AR 5312 (S34, E33 on 11 January, 1989) was a large sunspot group. Figure 1 shows a photospheric vector magnetogram obtained at 05:20 UT. A SF/M1.9 flare occurred at 06:26 UT; the maximum phase of the flare occurred at 06:49 UT. The flare ribbons are marked with thick closed lines which are based on the $\mathrm{H} \beta$ brightenings at 06:44 UT. It is interesting to note that although strong magnetic shear existed in many sections of neutral lines in this active region, the flare chose to occur in a potential neutral line. Figure 2 shows the profile of angular shear along the neutral line; the locus of the cut is marked with the thick dashed line shown in Figure 1. The beginning of the profile is marked by a cross, and the end is marked by an arrow. We will keep this convention for all the profiles throughout the paper. From the figures, we find that the angular shears are always smaller than $80^{\circ}$; in fact, more that $80 \%$ of pixels in the profile have shear smaller than $40^{\circ}$. It demonstrates that a flare can occur in neutral lines of somewhat lower shear.

In AR 6089 (N10, W10 on 10 June, 1990), a 2B/M2.3 flare occurred on at 06:05 UT. Figure 3 shows a vector magnetogram obtained at 06:03 UT; the peak of the flare is at 07:16 UT. The brightenings of the flare are drawn in Figure 3 with thick closed lines. Figure 4 shows the profile of angular shears along the neutral line. The angular shears remain high $\left(\geq 80^{\circ}\right)$ along the neutral line for more than $10000 \mathrm{~km}$. So this is a clear example that a flare occurs at a highly sheared neutral line.

We list some characteristics of all the 20 flares in Table I. Some symbols are defined as following: $\theta^{m}$, the maximum angular shear along the neutral line; $B_{t}^{m}$, 


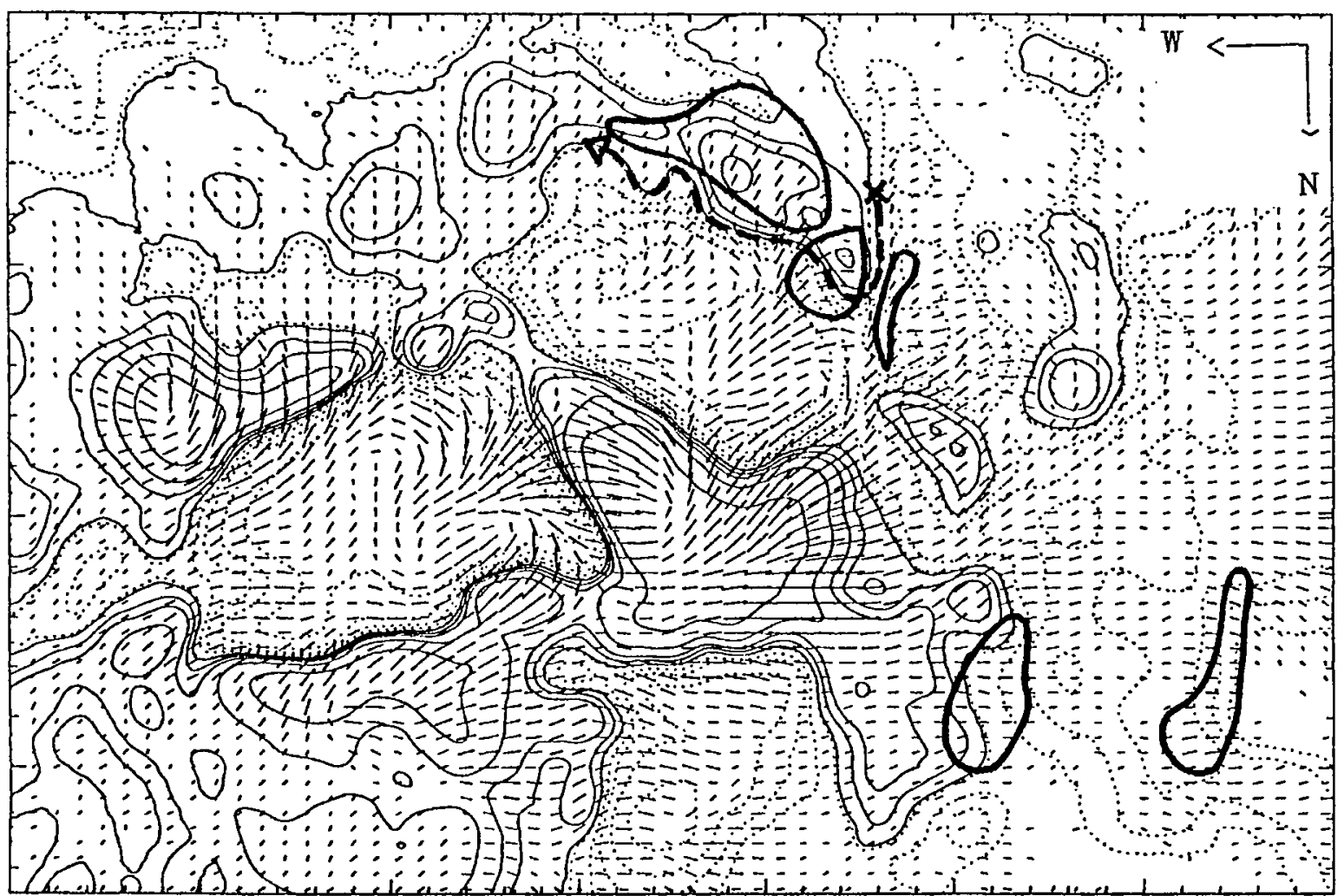

Fig. 1. The photospheric vector magnetogram of AR 5312 (S34, E33) obtained at 05:20 UT on 11 January, 1989. The field of view is $3.1 \times 4.7 \mathrm{arc}$ min. Solid and dashed contours represent positive and negative longitudinal magnetic fields. The lines represent the strength and direction of the transverse fields. The thick solid loops represent the positions of the flares at 06:44 UT. The thick dashed lines represent the neutral line.

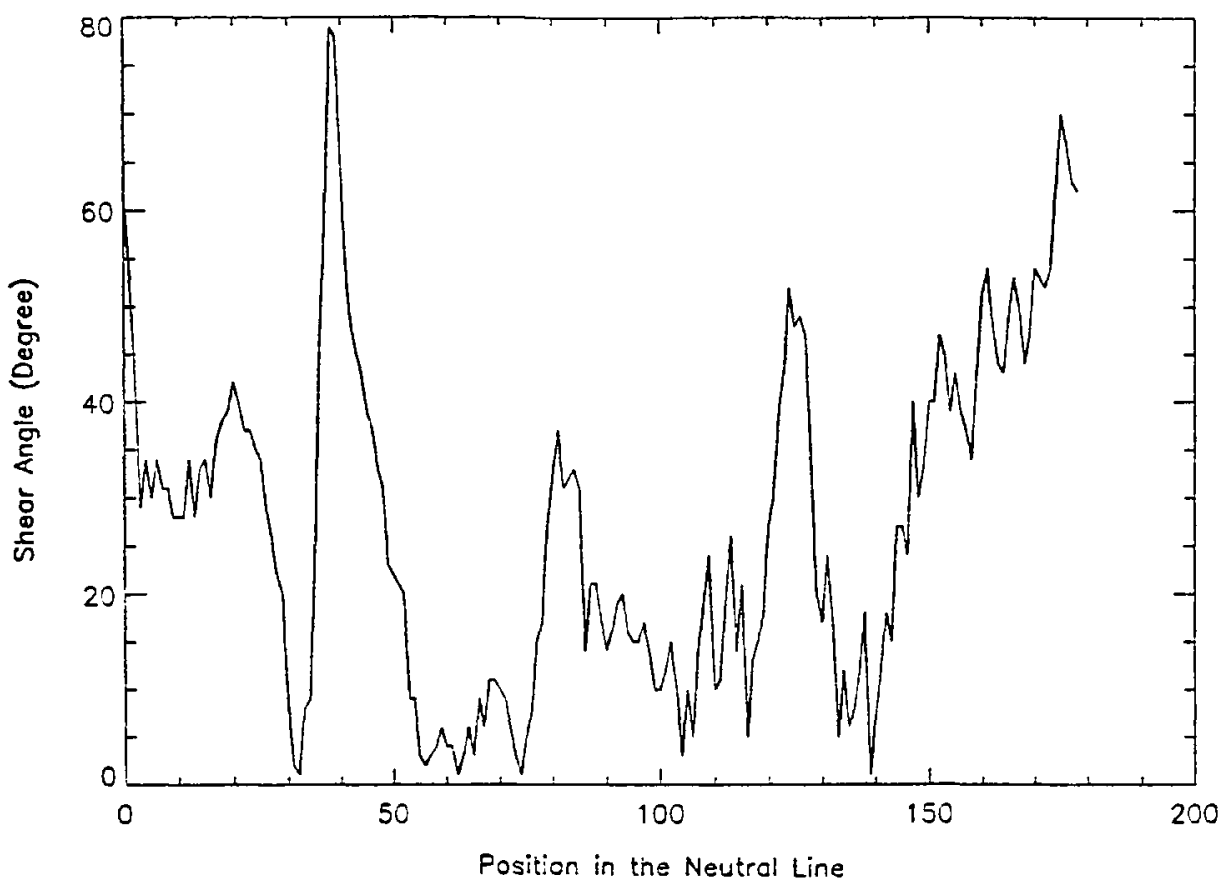

Fig. 2. The profile of angular shears along the neutral line of AR 5312. The beginning of the profile (zero coordinate) is marked by a cross in Figure 1, and the end is marked by an arrow. 


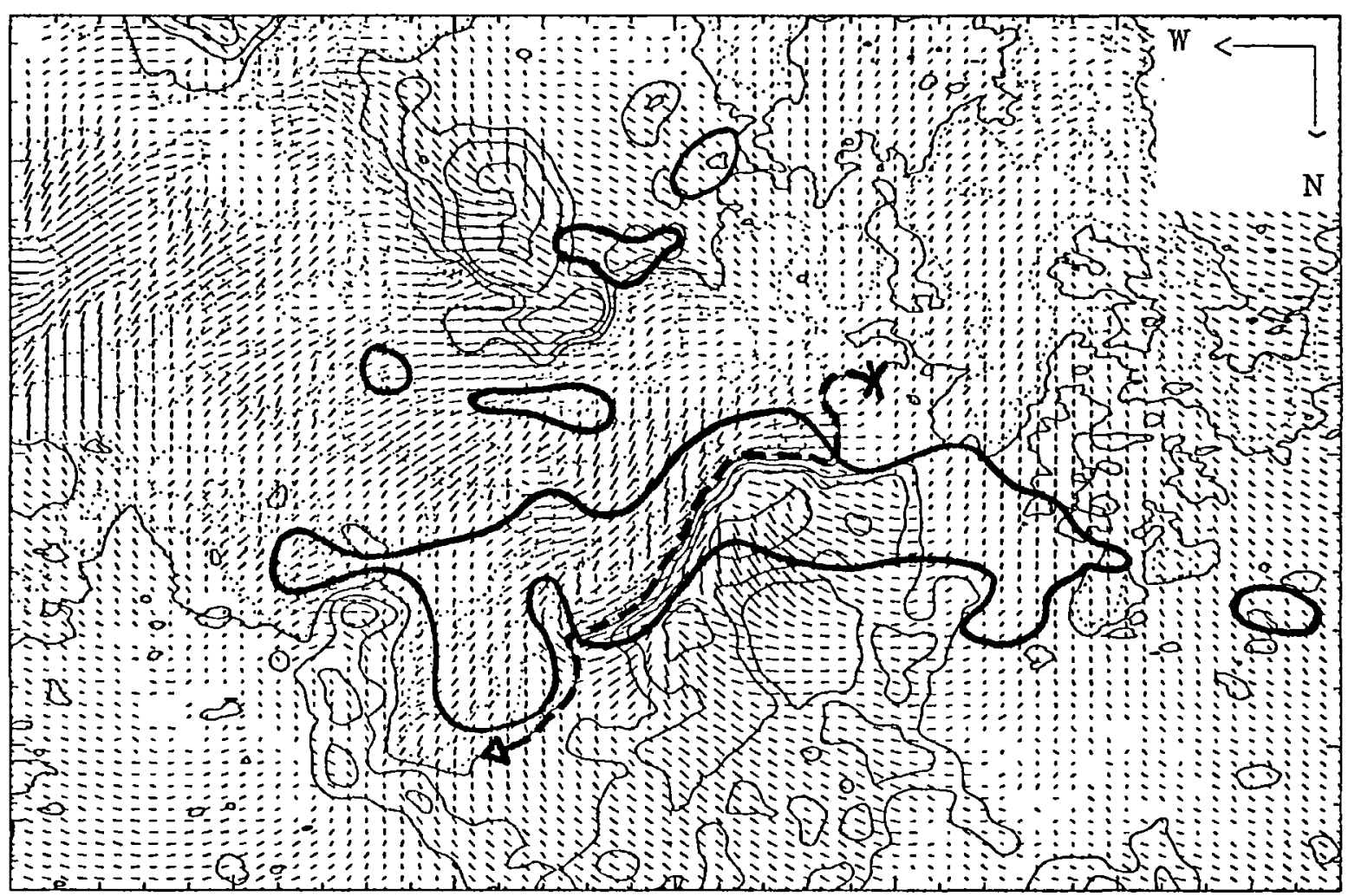

Fig. 3. The vector magnetogram of AR 6089 (N10, W10) obtained at 06:03UT on 10 June, 1990 with $2.3 \times 3.5$ arc min field-of-view.

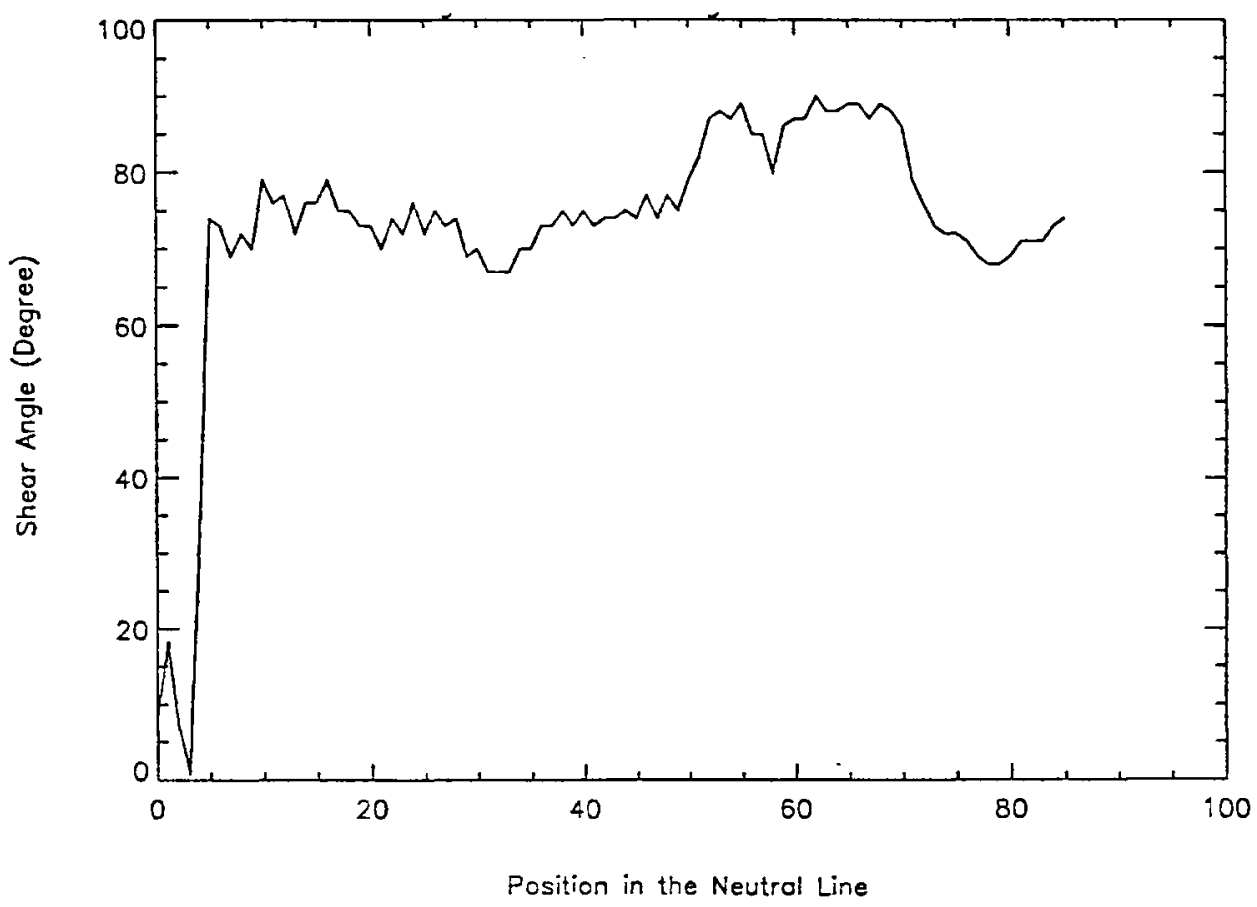

Fig. 4. The profile of angular shears along the neutral line of AR 6089. The beginning of the profile is marked by a cross in Figure 3, and the end is marked by an arrow. 


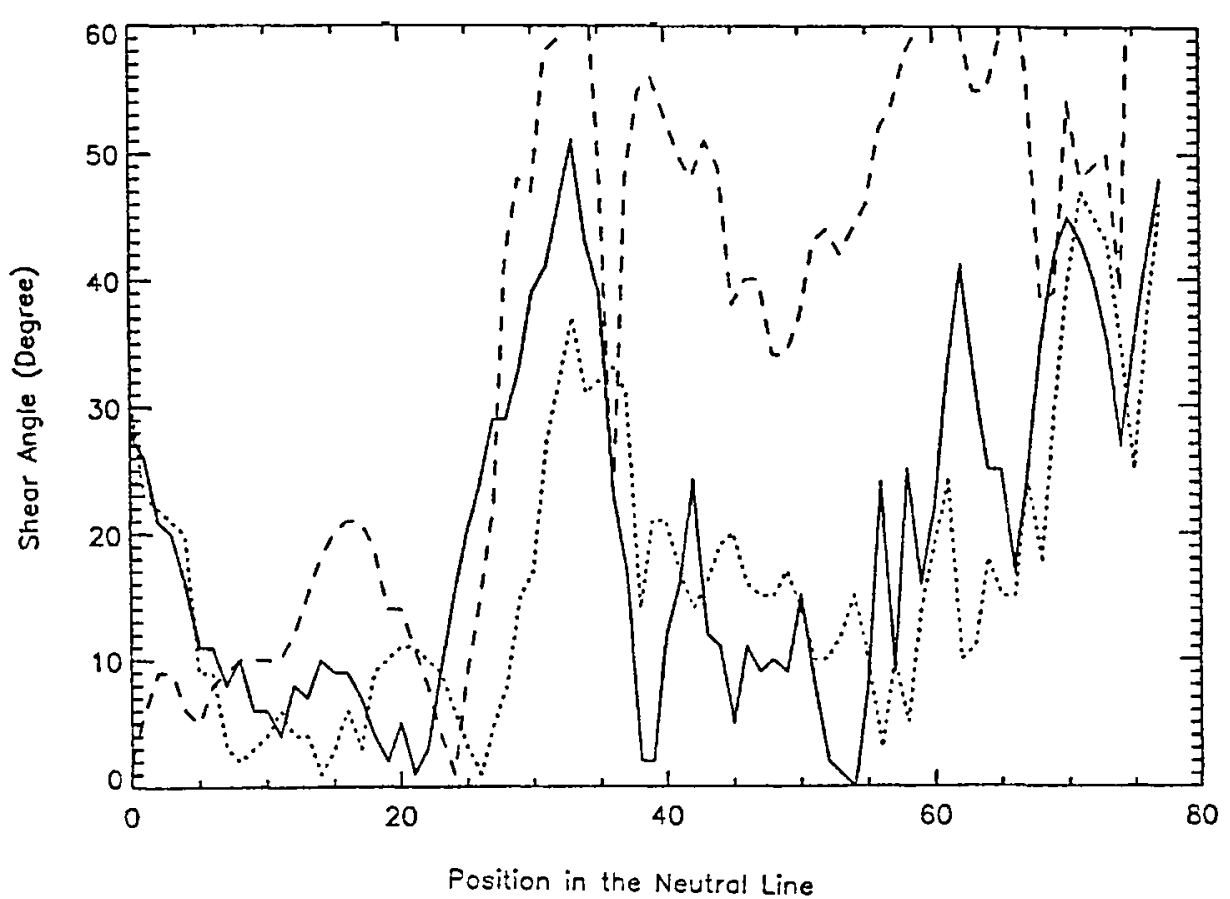

Fig. 5. The changes of angular shears along the neutral line of AR 5312 on 11 January, 1989. The solid line represents the profile of angular shears at 04:14 UT, the dotted line at 05:20 UT and the dashed line at 07:21 UT.

the maximum strength of transverse field along the neutral line; $\bar{\Theta}$, average angular shear along the neutral line; $\bar{B}_{t}$, average strength of transverse field along the neutral line (range of $B_{t}$ with $\theta \geq 80^{\circ}$ : the lowest and highest strengths of transverse field along the neutral line with the angular $\operatorname{shear} \theta \geq 80^{\circ}$ ); $L$, the length of the segments in the neutral line where the angular shear $\theta \geq 80^{\circ}$.

Among the 20 flares, 7 satisfy the high shear condition prescribed by Hagyard, so the occurrence of flares may not always require a highly sheared magnetic neutral line.

\subsection{Changes Before AND AFter THE Flares}

Since vector magnetograms are available before and after all the flares, changes of magnetic shear after the flare (if they exist) can be measured with our data. We now discuss two active regions, in which we do observe substantial changes of magnetic shear after three flares.

\subsubsection{The SF/M1.9 flare in AR 5312 (S34, E33 on 11 January, 1989)}

This SF/M1.9 flare began at 06:26 UT, peaked at 06:49 UT, and ended at 07:01 UT. Figure 1 shows a vector magnetogram obtained at 05:20 UT. Figure 5 shows the profile of angular shears along the neutral line (the thick line in Figure 1) before and after the flare. The shear angle averaged over the whole profile increased $13 \mathrm{deg}$ after the flare. 


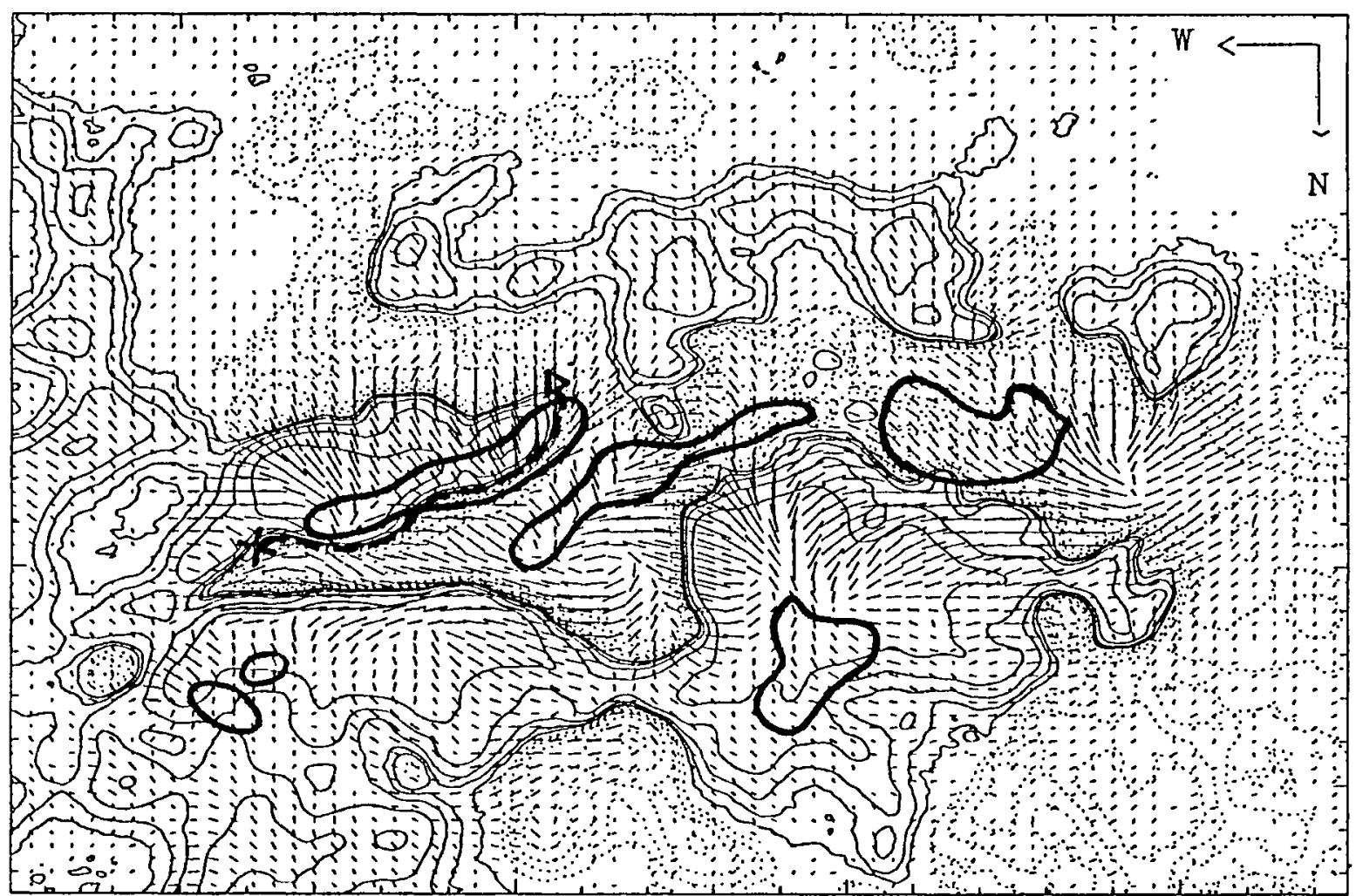

Fig. 6. The vector magnetogram of AR 5312 (S32, W10) obtained at 02:13 UT on 14 January, 1989 with a $4 \times 6$ arc min field-of-view.

3.2.2. The 1N/M6.1 and 2B/X2.1 flare in AR 5312 (S32, W14 on 14 January, 1989) An 1N/M6.2 and a 2B/X2.1 flares occurred on 14 January, 1989 within two hours. The first flare started at 02:08 UT and ended at 03:59 UT, and the second flare started at 04:05 UT and ended at 06:17 UT. Figure 6 shows the vector magnetogram obtained at 02:13 UT, and the thick contours show the $\mathrm{H} \beta$ brightenings of the $2 \mathrm{~B} / \mathrm{X} 2.1$ flare at its peak. The 1N/M6.2 flare occurred at the same general location. We obtained four vector magnetograms at 02:13, 04:33, 05:34, and 07:10 UT on 14 January. In Figure 6 we mark a thick dashed line along which the profiles of magnetic shear of the four magnetograms were calculated. Figure 7 shows the profiles of angular shears and Figure 8 plots the average angular shear along the neutral line as a function of time. It is obvious that the angular shears increase along the neutral line. The average angular shear increases $6.7 \mathrm{deg}$ after the flares.

In order to rule out the possibility that the observed changes of the shear in the above two cases are due to slight mis-alignment of the position of the neutral lines, we did the following test: we increase the width of the profile from 3 to 9 arc sec, by including 3 arc sec from each side in the neutral line. The shape of all the profiles remains the same. The increases of the shears are clearly shown in those new profiles. For both cases, the shape of the neutral line did not change after the flare; the increases of the shear are mainly due to the change of the azimuth in transverse fields. 


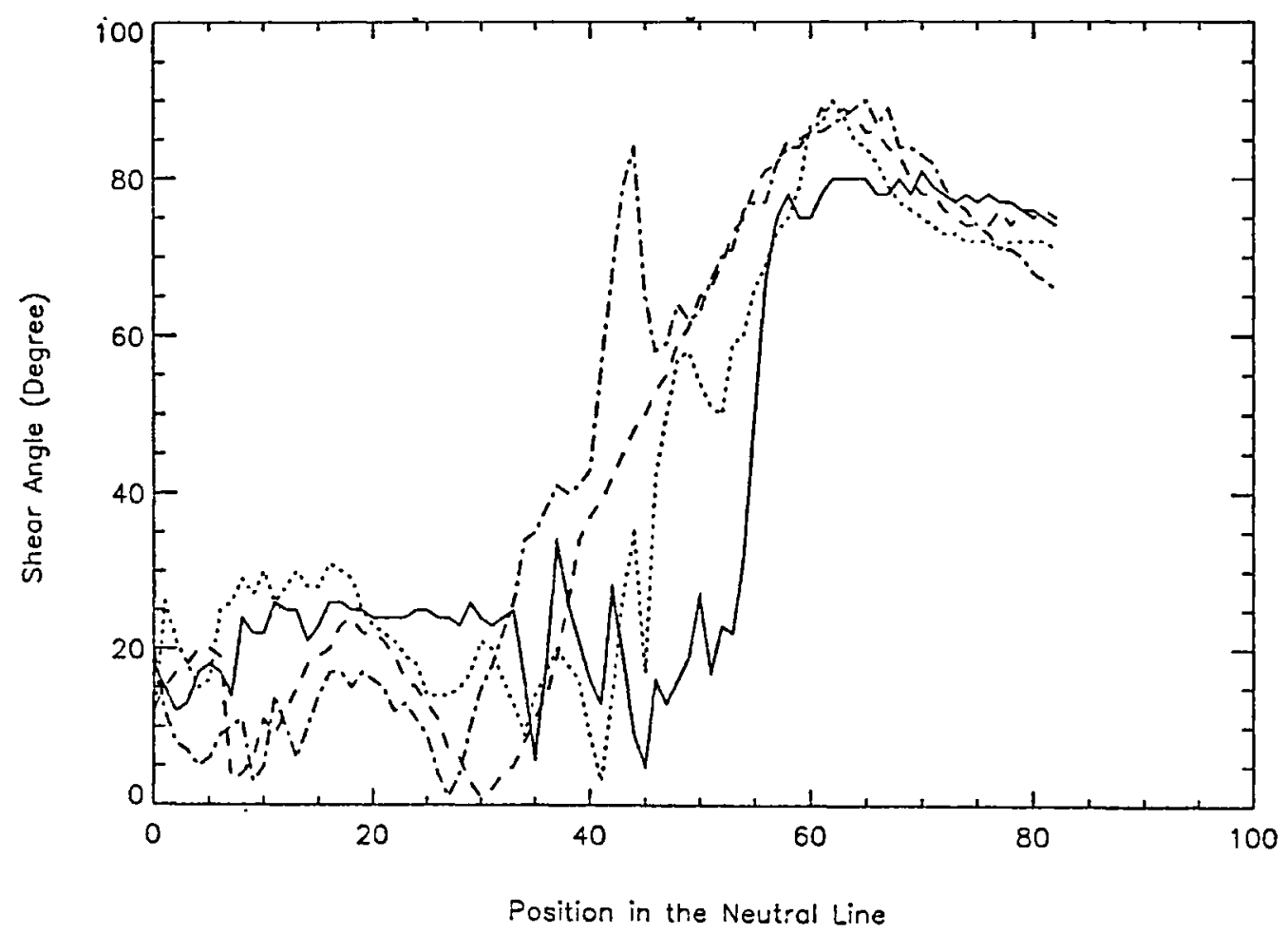

Fig. 7. The changes of angular shears along the neutral line of AR 5312 on 14 January, 1989. The solid lines are for the magnetogram observed at 02:13 UT; dotted lines, 04:33 UT; dashed lines, 05:34 UT; and dashed-dotted lines, 07:10 UT.

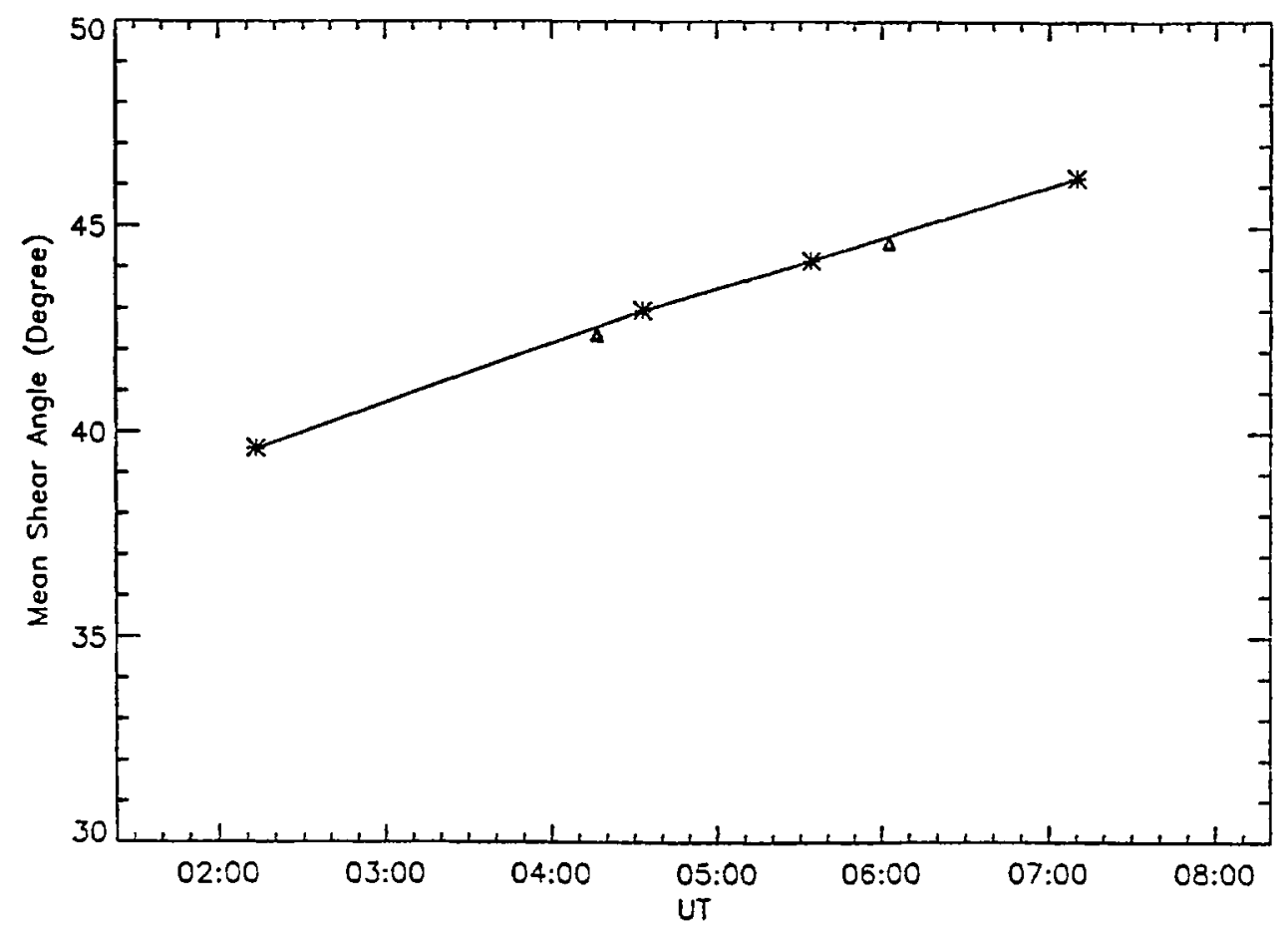

Fig. 8. The changes of average angular shears along the neutral line of AR 5312 on 14 January, 1989. The small triangles represent the beginning and the end of the $2 \mathrm{~B} / \mathrm{X} 2.1$ flare times. 
TABLE I

Characteristics of magnetic fields in flaring active regions

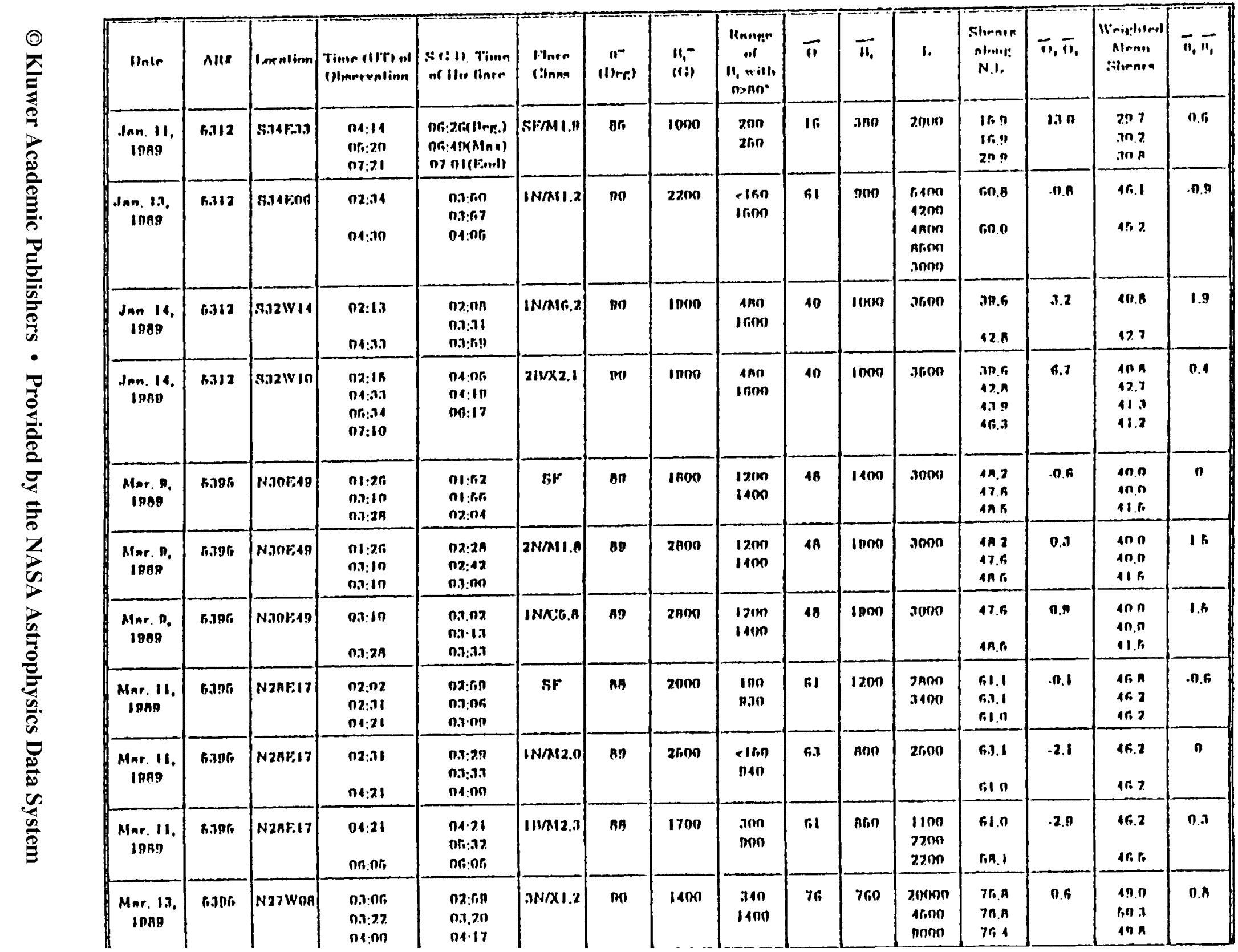


Table I (continued)

\begin{tabular}{|c|c|c|c|c|c|c|c|c|c|c|c|c|c|c|c|}
\hline lintr & All" & Iarenlinenl & 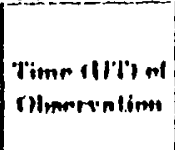 & 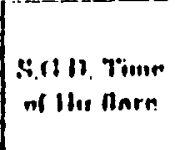 & $\begin{array}{l}\text { Pinner } \\
\text { (inmen }\end{array}$ & (Irrn) & $\begin{array}{l}\text { is:- } \\
\text { (ii) }\end{array}$ & 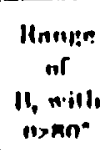 & $\bar{"}$ & $\overrightarrow{u_{0}}$ & I. & $\begin{array}{l}\text { Silenner } \\
\text { ninuge } \\
\text { N.8. }\end{array}$ & $\overline{n_{1}} \bar{n}_{1}$ & 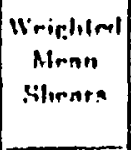 & $\overline{n_{1}} n_{1}$ \\
\hline $\begin{array}{c}\text { Angr. } 14, \\
\text { inan }\end{array}$ & מ.jn & N27W22 & $\begin{array}{l}08: 01 \\
\text { m:2n: }\end{array}$ & $\begin{array}{l}\text { nf: } 2 n \\
\text { nar:2n } \\
\text { nor:an }\end{array}$ & $\mathrm{SN}$ & mn & 145,in & $\begin{array}{l}2,00 \\
131010\end{array}$ & $7 n$ & $71 \times n$ & 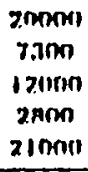 & $\begin{array}{l}77.6 \\
77.1\end{array}$ & . 7.6 & $\begin{array}{l}\text { 10.7.1 } \\
\text { B.4.1. }\end{array}$ & 1.4 \\
\hline $\begin{array}{l}\text { Alar. 14. } \\
\text { inn? }\end{array}$ & 6.396 & N27W22 & $\begin{array}{l}\text { ma;pro } \\
\text { n7:10 } \\
\text { n8:1n }\end{array}$ & $\begin{array}{l}\text { n6:1n } \\
\text { n6:27. } \\
\text { n7:1n }\end{array}$ & SNAR.A & 100 & ition & 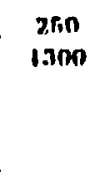 & 78 & $7 m$ & $\begin{array}{c}20000 \\
7.30 n \\
17.0011 \\
2 A m n \\
2100 n\end{array}$ & $\begin{array}{l}77.5 \\
717.7 \\
73.4\end{array}$ & 2.1 & 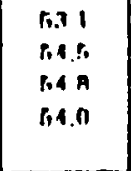 & 1.7 \\
\hline $\begin{array}{c}\text { Mhar } 70, \\
\operatorname{lng} 0\end{array}$ & 6061 & SIGWII & $\begin{array}{l}01: 2 n \\
02: 16 \\
n 3: 62 \\
04: 16 n\end{array}$ & $\begin{array}{l}n, 7: 19 \\
0.3: 10 \\
06: 16\end{array}$ & IN/All. & 74 & RON & & 13 & 220 & & $\begin{array}{l}.7 .1 .1 \\
.37 .1 \\
.36 .3\end{array}$ & $.1 . n$ & $\begin{array}{l}.72 .7 \\
36.6 \\
x n .8 \\
31.0\end{array}$ & .1 .5 \\
\hline $\begin{array}{c}\text { Ang } 20 . \\
\operatorname{lagno}\end{array}$ & Fon. & N.נ. & $\begin{array}{l}\text { 22:5ro } \\
\text { n2:m }\end{array}$ & $\begin{array}{l}22 \cdot 4 n \\
22: 11 \\
n 1: n n\end{array}$ & 2F/MU1.6 & $m$ & 1100 & $\begin{array}{c}7.3 n \\
11900\end{array}$ & 73 & TAD & 22000 & $\begin{array}{l}72.7 \\
\text { fin s. }\end{array}$ & .4 .1 & $\begin{array}{l}46.6 \\
46.3\end{array}$ & .0 .2 \\
\hline 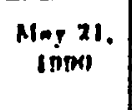 & ROA.1 & N.1.7W.1.10 & $\begin{array}{l}m 0: 17 \\
n 2: m\end{array}$ & $\begin{array}{l}n 1: 21 \\
n 1: 2 n \\
n 2: n r\end{array}$ & 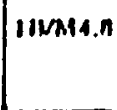 & 80 & 17001 & $\begin{array}{l}3.010 \\
470\end{array}$ & $4 \pi$ & reno & A.Xom & $\begin{array}{l}41.8 \\
11.1\end{array}$ & .7. A & $\begin{array}{l}11.9 \\
4.1 .1\end{array}$ & 1.0 \\
\hline $\begin{array}{c}\text { Mnr 21. } \\
10 n 0\end{array}$ & Rins, & M.,.3W ח ח & $\begin{array}{l}\text { D6:0n } \\
07: 22\end{array}$ & $\begin{array}{l}n n_{0}: 12 \\
m r: 14 \\
n 7: 22\end{array}$ & $2 \mathrm{~N} / \pi$ & ma & $\begin{array}{l}21,010 \\
.740\end{array}$ & 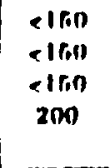 & $\begin{array}{l}71 \\
71\end{array}$ & $\begin{array}{l}\text { eiron } \\
\text { eiron }\end{array}$ & 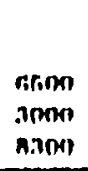 & & & $\begin{array}{l}4.17 \\
4.12\end{array}$ & 1.3 \\
\hline $\begin{array}{c}\text { Niny 22. } \\
\text { IDAB }\end{array}$ & nons & s10W14 & $\begin{array}{l}\text { 2.3:24 } \\
\text { n2:19 }\end{array}$ & $\begin{array}{l}m, n d \\
m ;: 1 A \\
n 2: n 1\end{array}$ & IN/M.7.2 & 69 & 1000 & & 18 & Bro & & $\begin{array}{r}8 n .1 \\
10.7 \\
\end{array}$ & 1.1 & $\begin{array}{r}28.0 \\
. \quad 11.32 \\
\end{array}$ & 2.1 \\
\hline 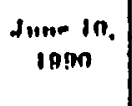 & Gonn & Ninivin & $\begin{array}{l}\text { Of:01 } \\
\text { on:2n }\end{array}$ & 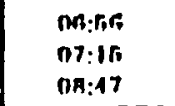 & $211 / 1212.3$ & mo & nMT) & $\begin{array}{l}\ln 00 \\
\ln 00\end{array}$ & 77 & 140 & I IRPQPI & $\begin{array}{r}76.6 \\
7 n .4 \\
\end{array}$ & 1.0 & $\begin{array}{r}12.2 \\
44 . \\
\end{array}$ & 2.2 \\
\hline $\begin{array}{c}3 m-12 . \\
1090\end{array}$ & Fong & NinW:3. & $\begin{array}{l}2.1: 17 \\
07: 30\end{array}$ & $\begin{array}{l}04: 28 \\
04: 01 \\
n r: 1.1\end{array}$ & ZIM:r.z & nn & 1100 & $\begin{array}{l}2150 \\
\text { R60 }\end{array}$ & fir & 300 & 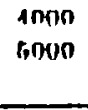 & & & & \\
\hline
\end{tabular}


We use the same analysis methods for all the 20 flares in the 6 active regions. In addition, we also measured the mean weighted shear angles in a small rectangular region covering the flare ribbons. In Table I, we list our results. Some parameters are defined as following:

$\bar{\Theta}_{2}-\bar{\Theta}_{1}$, the change of average angular shears along the neutral lines before and after the flare.

$\bar{\theta}_{2}-\bar{\theta}_{1}$, the change of the mean weighted shear angles in the rectangular region covering the flare ribbons.

Besides the two cases discussed above we do not detect substantial shear changes associated with flares. Even for these two cases, the increase of the mean weighted shear angles is much smaller than the increase of the shear angle at the neutral lines. This confirms the result of Wang et al. (1994), that the shear change concentrates in a band near the neutral line.

\subsection{THE POSITIONS OF INITIAL BRIGHTENINGS}

Tang and Wang (private communication) found that if initial brightening of a flare is close to the neutral line, shears (defined by both the shear angle and transverse field strength) are usually strong at the neutral line; if the initial brightenings are further away from the neutral line, the shears are weaker. Our data give partial confirmation to this result.

In AR 5395 (N28, E17) on 11 March, 1989, a SF flare began at 02:58 UT (by brightness of $\mathrm{H} \beta$ ); in Figure 9, we draw the positions of initial brightenings (thick solid contours) and the flaring neutral line (thick dashed lines) on a vector magnetogram obtained at 02:02 UT. We can see that most of the points are near the neutral lines where the transverse field strength is close to $1000 \mathrm{G}$ and the mean sheared angle along the neutral line is $61^{\circ}$ (Table I).

In AR 6891 (S14, E22), a 3B flare occurred on 27 October, 1991, beginning at 05:34 UT (by brightness of $\mathrm{H} \beta$ ). Figure 10 is a vector magnetogram obtained at 05:01 UT. Again the initial brightenings and flaring neutral line are drawn by thick solid contours and dashed lines respectively. We selected this neutral line because the the post-flare loops were crossing it. The transverse field strength is below $150 \mathrm{G}$ along the whole neutral line. The shear angles can not be determined because the field strength is below our sensitivity limit. It is obvious that most of initial brightenings are 30 arc sec away from the neutral lines. The neutral line associated with this flare does not have strong shear because of its low transverse field strength. 


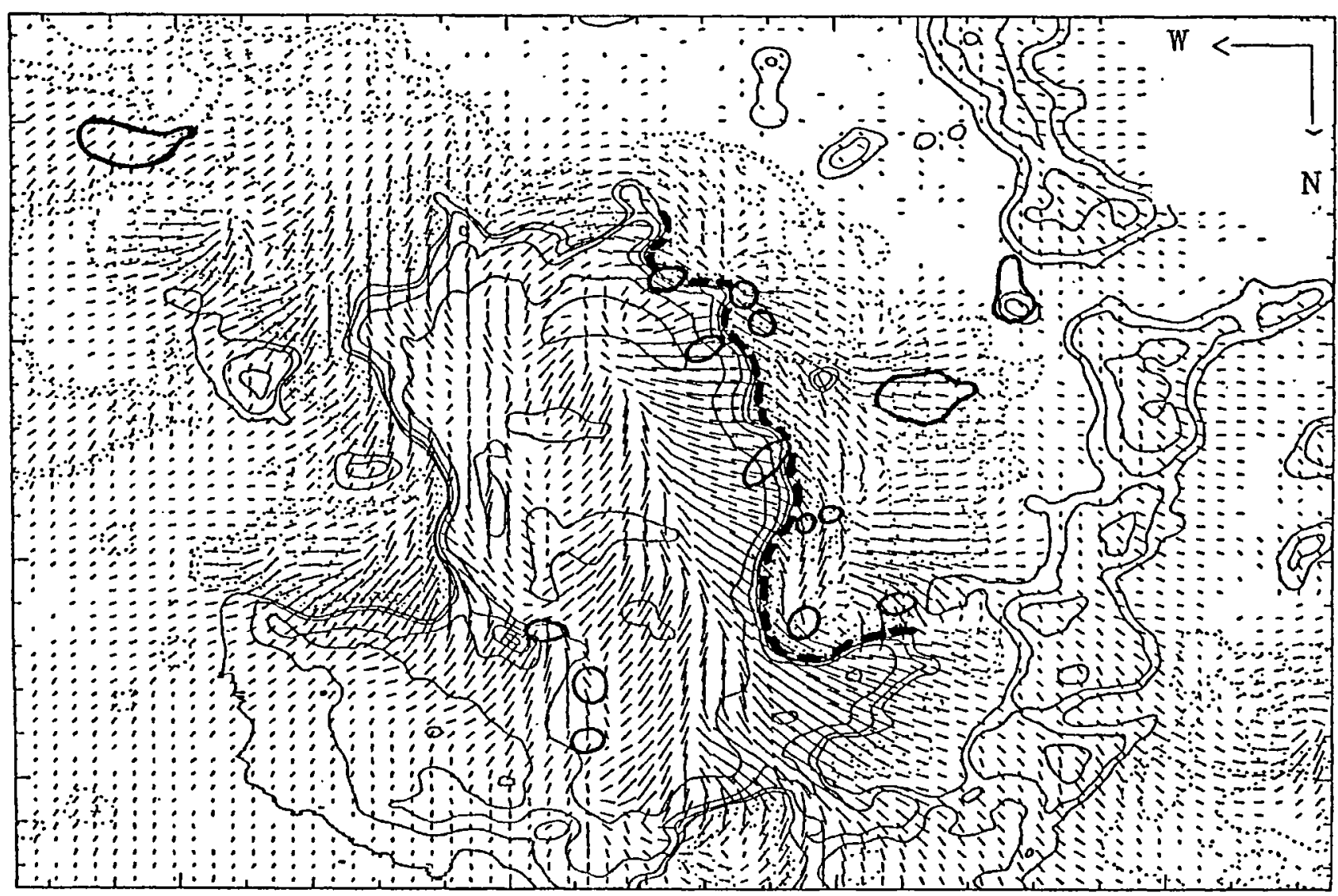

Fig. 9. The vector magnetogram of AR 5395 (N28, E17) obtained at 02:02 UT on 11 March, 1989 with a $3.1 \times 4.7$ arc min field-of-view.

\section{Summary and Discussion}

We summarize our results as following:

(1) Among the 20 major flares (including two X-class flares), 7 satisfy the flare conditions prescribed by Hagyard. The average shear angle of all the flaring neutral lines was $56^{\circ}$ (above $45^{\circ}$ ), so we conclude that flares prefer to occur in more sheared neutral lines. However, strong photospheric shear $\left(\geq 80^{\circ}\right)$ along the neutral line is not necessarily a condition for a flare. Nine flares occur at potential neutral lines with mean shear $<50^{\circ}$. Transverse field strength of the flaring neutral lines ranged from $<100 \mathrm{G}$ to $2800 \mathrm{G}$.

(2) Based on very limited cases where we can track the initial brightenings of the flares, we confirm the result found by Tang and Wang: if the initial brightenings are close to the neutral line, then the neutral line tends to have higher shear; and if the initial brightenings are away from the neutral line, the neutral line tends to be less sheared.

(3) Eighty percent of the initial brightenings occur in the most sheared sections of a neutral line, but there are many exceptions.

(4) A 6-deg shear increase along the neutral line after an X-2 flare was detected. A 13-deg increase after a M-1.9 flare was also detected. For other flares, the changes 


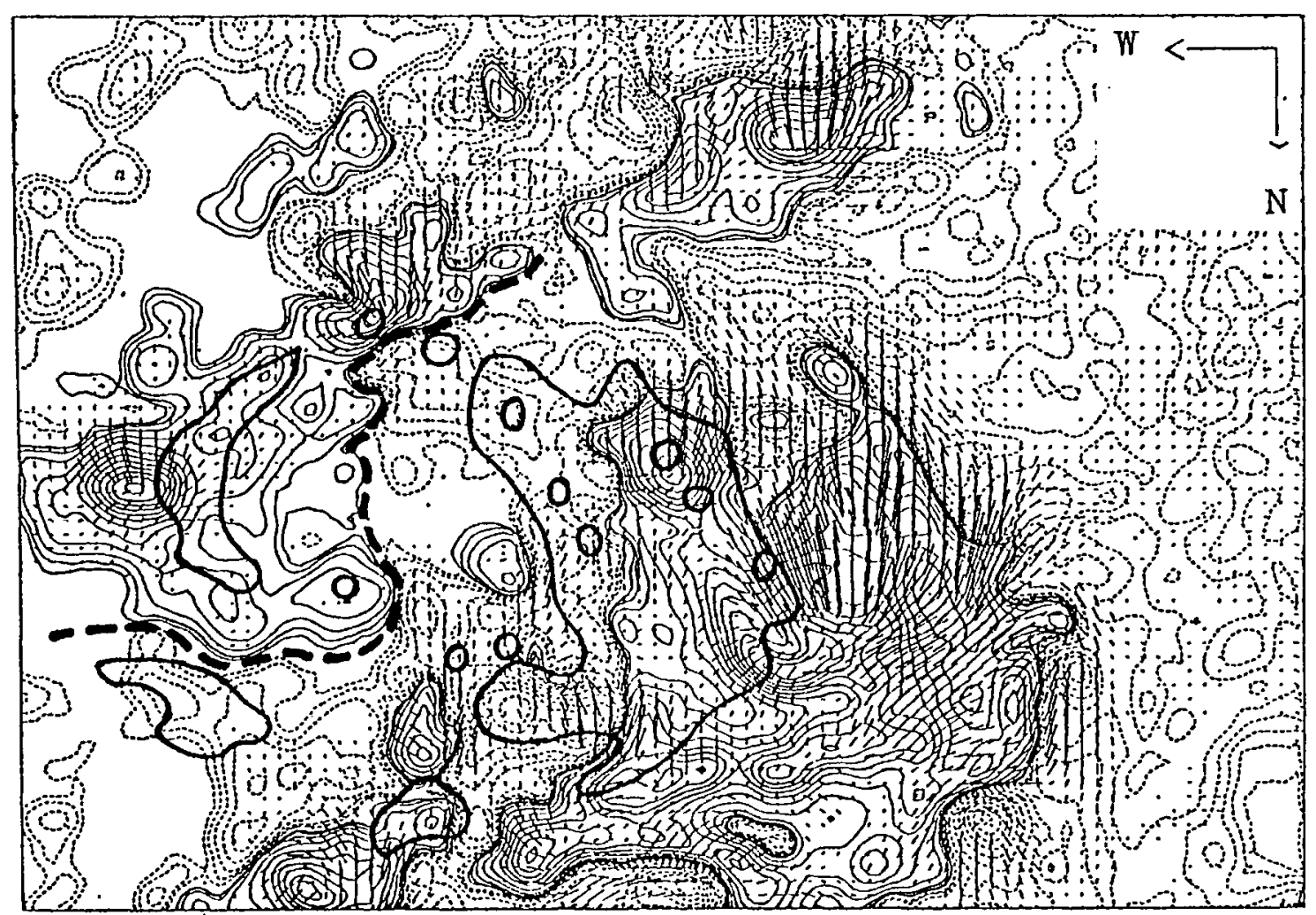

Fig. 10. The vector magnetogram of AR 6891 (S14, E22) obtained at 03:28 UT on 27 October 1991 with a $4 \times 6$ arc min field-of-view.

are within the noise level; about half of them show an increase and other half a decrease. We also calculated the mean weighted shear angles in the flaring regions, and did not find the changes to be above the noise level. The shear increase after flares (Wang et al., 1994) may be only associated with large X-class flares.

It is generally accepted that magnetic reconnections occur above the photosphere, so the magnetic fields there may be sheared to cause the flare. It is not necessary to require that the magnetic field is also sheared in the photosphere level. We also note that many flares were associated with the emergence of new flux at the time of the flares; this confirms the result of Wang and Tang (1993).

\section{Acknowledgements}

We thank Mr Marc Tamsky for helpful discussions. The study was supported by NSF grants INT-9206863 and ATM-9122023, by NASA grant NAGW-1972, and by ONR grant N00014-89-J-1069. HW acknowledges support from NASA grant NAG5-2090.

\section{References}


Hagyard, M. J., Smith, J. B., Jr., Teuber, D., and West, E. A.: 1984, Solar Phys. 91, 115. Wang, H.: 1992, Solar Phys. 140, 85.

Wang, H. and Tang, F.: 1993, Astrophys. J. 407, L89.

Wang, H., Ewell, M.W., Zirin, H. and and Ai, G.: 1994, Astrophys. J. 424, 436. 